OPEN ACCESS

Edited by:

Daphne Economou, University of Westminster,

United Kingdom

Reviewed by:

Darryl Charles,

Ulster University, United Kingdom

Christopher M. Laine,

University of Southern California,

United States

${ }^{*}$ Correspondence:

Matthew Schmidt

matthew.schmidt@coe.ufl.edu

Specialty section:

This article was submitted to Virtual Reality in Medicine, a section of the journal

Frontiers in Virtual Reality

Received: 29 September 2020 Accepted: 28 January 2021 Published: 19 March 2021

Citation: Schmidt M, Newbutt N, Schmidt C and Glaser N (2021) A Process-Model for Minimizing Adverse Effects when Using Head Mounted Display-Based Virtual

Reality for Individuals with Autism. Front. Virtual Real. 2:611740. doi: 10.3389/frvir.2021.611740

\section{A Process-Model for Minimizing Adverse Effects when Using Head Mounted Display-Based Virtual Reality for Individuals with Autism}

\author{
Matthew Schmidt ${ }^{1 *}$, Nigel Newbutt ${ }^{1}$, Carla Schmidt ${ }^{1}$ and Noah Glaser ${ }^{2}$ \\ ${ }^{1}$ Educational Technology Program, University of Florida, Gainesville, FL, United States, ${ }^{2}$ Educational Psychology, University of \\ Connecticut, Storrs, CT, United States
}

Interest in the use of virtual reality technologies for individuals with autism spectrum disorders has been increasing for over two decades. Recently, research interest has been growing in the area of head mounted display-based virtual reality technologies, thanks to increased availability and affordability. Affordances and theorized benefits of headsetbased virtual reality for individuals with autism spectrum disorders are quite promising. However, very little attention has been given in the literature to implementation safety and ethics. This is a particular concern in light of documented adverse effects associated with headset-based virtual reality. To approach this gap, this article details how the authors approached the issue of minimizing adverse effects with related and overlapping methods, but from two separate, independent research sites - one in the United States and one in the United Kingdom. A structured within- and across-case analysis of the two independent studies was conducted to identify central implementation processes and procedures. Analysis resulted in development of a model for minimizing potential adverse effects of headset-based virtual reality for this population. We assert that our model could provide clarity in terms of design and implementation of headset-based virtual reality for individuals with autism spectrum disorders, guide implementations of future researchers and practitioners, and contribute to minimizing and controlling for potential adverse effects.

Keywords: autism, virtual reality, head-mounted displays, implementation, adverse effects, cybersickness

\section{INTRODUCTION}

Researchers have been exploring the use of head-mounted displays (HMDs) and virtual reality (VR) in the area of autism spectrum disorders (ASD) since Strickland (1996) seminal work over two decades ago. Recently, interest and research activity have been increasing substantially due to the availability of affordable, consumer-grade HMD-based VR systems. ASD is a lifelong condition characterized by challenges with social communication/interaction and restricted, repetitive patterns of behavior (American Psychiatric Association, 2013; DSM-5 American Psychiatric Association, 2013). Manifestations of ASD present substantial heterogeneity across affected individuals (Masi et al., 2017). Early intervention has been shown to lead to improvements in core autism symptoms (Estes et al., 2015). However, access to and consistency of services in early intervention are known barriers, potentially leading to challenges in adulthood such as chronic underemployment, social isolation, and inability to live independently (Eaves and Ho, 2008; Taylor 
and Seltzer, 2011; Taylor and Mailick, 2014; Hedley et al., 2017). Prevalence of autism is increasing, with current estimates suggesting one in 54 children receiving an ASD diagnosis in the United States alone (Russell et al., 2014). As such, viable and effective therapeutic interventions are in high demand. Virtual reality has garnered significant attention as a potentially effective solution for delivering such interventions (Bellani et al., 2011; Parsons, 2016).

Virtual reality is considered to hold tremendous promise for individuals with ASD due to: (1) the suitability and natural affinity to computers and technology for this population; (2) the ability of HMD-based VR to simulate real-world situations and contexts in safe and predictable ways; and (3) the ability to control, shape, and tailor VR interventions to participant needs and local contexts (Parsons, 2016; Bozgeyikli et al., 2018). Therefore, VR appears to be intrinsically reinforcing for individuals with ASD, has technological affordances which align closely with instructional needs, and can broaden access to a range of services (Parsons et al., 2020). VR provides individuals with ASD the ability to encounter and practice skills in highly realistic and customizable contexts, for input stimuli to be intentionally manipulated, and to have real-world consequences mitigated or removed. Within this context, a patchwork of research studies have investigated VR-based interventions across multiple domains such as social/emotional skills (Moore et al., 2005; Ke and Im, 2013), safety skills (Self et al., 2007), street-crossing (Josman et al., 2011), daily living (Parsons et al., 2004; Mitchell et al., 2007; Jarrold et al., 2013), and communication (Kandalaft et al., 2013) to name but a few (Wang and Anagnostou, 2014; Mesa-Gresa et al., 2018). While reported research outcomes in this area suggest VR has promise for individuals with ASD, as a whole, research supporting connections between theorized benefits of VR HMDs and empirical evidence supporting effectiveness has been described as limited, piecemeal, and largely inconclusive (Parsons, 2016).

To date, the majority of research has focused on the use of desktop-based VR and virtual worlds. More recently, research interest in HMD-based VR for individuals with ASD has increased, in part due to increased consumer availability of HMDs. Researchers are particularly interested in implementing HMD-based VR for individuals with ASD, as specific affordances of the technology such as heightened sense of presence, immersion and ecological validity all lend themselves to a meaningful environment that potentially can enable opportunities for acquisition, maintenance, and generalization of skills (Freina and Ott, 2015). However, a widely-acknowledged concern related to using HMD-based VR is the potential that users will experience some degree of adverse effects (Palmisano et al., 2017). By adverse effects, we refer to negative effects including cybersickness (e.g., nausea, eye-strain, etc.), safety concerns (e.g., stumbling and falling), increased anxiety, sensory disturbances, etc. (cf. Kellmeyer, 2018). Although an abundance of calls for future research to investigate adverse effects can be found in the literature (e.g., Irish, 2013; Fletcher-Watson, 2014; Wong et al., 2015; Mesa-Gresa et al., 2018), actual research on adverse effects is largely absent. Research in the field has yet to meaningfully and systematically approach questions related to adverse effects for individuals with ASD when applying VR in general, and HMDbased VR specifically (Bradley and Newbutt, 2018; Malihi et al., 2020).

The majority of research on VR interventions for individuals with ASD has been principally concerned with establishing intervention effects. However, researchers' desire to establish intervention effects problematically positions VR technology as the primary driver of intervention outcomes. To-date, no design heuristics or guidelines have been published in this area. Although a variety of heuristics and design principles exist for developing 2D computer interfaces for individuals with ASD (Benton et al., 2011; Khowaja and Salim, 2013), far less is known about how to design 3D interfaces and environments: "[...] there is no well-established literature on the best practices in designing VR user interface attributes for individuals with ASD yet" (Bozgeyikli et al., 2018, p. 22:5). With a dearth of guidance in terms of VR design best practices for individuals with ASD, researchers are broadly left to their own devices when confronting how to design effective interventions. The lack of practical, detailed, and considered processes to help minimize possible adverse effects in published research suggests a gap in the literature that could limit the full potential of HMD-based VR for individuals with ASD.

Alongside the lack of guidance for designing 3D interfaces and environments for individuals with ASD in general, there is also a lack of understanding regarding how these interfaces and environments might lead to potential adverse effects when experienced in HMDs specifically. That adverse effects have not been a particular focus within the field poses a potential issue around researchers adopting and using emergent technologies like HMD-based VR before their implications of use are understood or before evidence-based recommendations for implementation are established. Borrowing from Kellmeyer (2018), "very little systematic discussion of the neurophilosophical and ethical challenges from the clinical use of these new VR systems is available" (p. 2). Given the limited research in the field, a significant gap exists related to exploring and reporting potential adverse effects (Bradley and Newbutt, 2018). We argue that with rapid technological advancements in this area, researchers, practitioners, and other professionals working with people with ASD have an urgent responsibility to confront these issues (Parsons, 2016).

Thus far we have established the three following research gaps: (1) consideration of adverse effects of VR for individuals with ASD in published research is limited; (2) published design heuristics and principles related to VR for individuals with ASD are largely absent; and (3) there is a paucity of research that explicitly explores and reports potential adverse effects for this vulnerable population. The purpose of this article, therefore, is to approach these gaps by offering a set of preliminary implementation guidelines for HMD-based VR to minimize potential adverse effects for individuals with ASD. Our work is intended to guide researchers and practitioners alike, and therefore serves to bridge a research-to-practice gap in this area.

We turn to our own practice to assert our design expertise in this area, establish our positionality, and frame the issue from the 
perspective of expert recommendations and best practices, as informed by ethical guidelines. We, the authors, are among the first to have pursued formal research studies with HMD-based VR for individuals with ASD. Our research was performed in two separate, independent research groups. We have published and presented widely on VR for ASD and iteratively have refined our implementations (e.g., Schmidt et al., 2012; Newbutt et al., 2016; Newbutt et al., 2017; Glaser and Schmidt, 2018; Newbutt, 2019; Schmidt et al., 2019; Newbutt et al., 2020). Over the course of multiple studies and through working with stakeholders, we have developed principles and expert knowledge that inform our professional practice This has been achieved through a process of reflection-on-action that considers a range of critical issues and factors directly affecting the acceptance, perceived utility, implementation quality, and participant well-being of VR experiences for this population.

\section{MATERIALS AND METHODOLOGY}

This article seeks to address a research-to-practice gap of insufficient implementation guidelines for minimizing potential adverse effects of using HMD-based VR for individuals with ASD. Our specific aims are (a) to explore how two independent research teams sought to minimize adverse effects for research participants in two separate studies and (b) to distill the lessons learned from case comparison into actionable guidelines that might be used by researchers and practitioners in their local contexts. On this basis, and alongside the gaps in knowledge presented previously, the following research questions guided our inquiry:

1. What are the common elements and/or key points of differentiation across studies?

2. What themes emerge from comparison of the individual studies performed at Study 1 and Study 2?

3. What lessons can be drawn from within- and across-case comparison related to minimizing adverse effects of HMDbased VR for individuals with ASD?

To approach these questions, we analyzed two recently published research studies (Newbutt et al., 2020), associated artifacts, and documentation so as to extract and synthesize salient details of how researchers implemented HMD-based VR in their studies. The first and second authors were the lead researchers. Case study methodology was used to perform a structured within- and across-case analysis of implementation procedures with the goal of promoting analytic generalizations (Yin, 2017). A "case" was defined as one of the two independent research studies led by the first and second author.

The USA-based study led by the first author (Schmidt et al., 2019) utilized a design-based research (DBR) methodological approach that considered ease-of-use, the nature of participants' user experience, feasibility, and relevance of a spherical, videobased virtual reality (SVVR) app as well as a fully immersive, collaborative $3 \mathrm{D}$ virtual environment, both of which were delivered using HMDs for a group of adults with ASD in a day program. The UK-based study led by the second author (Newbutt et al., 2020) sought to place children on the autism spectrum at the center of a study examining the potential of VR HMDs used in primary and secondary school classrooms; explored a range of VR experiences, how autistic users reported physical experiences, enjoyment, and potential of VR HMDs in their classrooms, while systematically exploring potential adverse effects.

Our work is presented as an instrumental, collective case study (Thomas, 2015). Our analytic process involved describing each case in detail and then presenting themes within the case, followed by thematic analysis across cases. As articulated in Figure 1, we adopted a three-phase approach. Phase 1 consisted of structured critical discussions in which we reviewed and summarized research details, methods, and findings, and established a basis for across-case comparison. In phase 2, artifacts were systematically discussed and reflected upon through formal meetings in which authors interrogated one another's work to identify themes. Finally we synthesized lessons learned (Yin, 2017) into a framework that captured best practices.

\section{Trustworthiness}

We implemented a range of techniques to ensure the methodological rigor of our research from Lincoln and Guba (1985) recommendations for promoting trustworthiness. Most important to trustworthiness is establishing credibility (Shenton, 2004), which we sought to accomplish in the current case study in three ways. We aimed to promote transferability, which according to Tobin and Begley (2004), refers to the generalizability of research from one case to another. Thick description of our separate contexts is provided in the results of phase 1 below. By presenting similar findings from two studies, but in two separate settings, we provide a more inclusive overall picture. We also aimed to establish dependability and confirmability by maintaining an audit trail. We provide in the results section below a clear and logical description of our research process. We report our process in detail so that it can be repeated in future work. In this way, readers could adopt the research design we report here as a type of prototype model (Shenton, 2004). To allow the traceability of our work in a stage-wise process, we used software that logged all changes to our work over time. Our audit trail consists of versioned files that include step-by-step histories of the methods and findings presented in this paper.

\section{RESULTS}

In the following sections, we present the outcomes of our withinand across-case analysis according to three separate phases of analysis. We present these phases in alignment with our research questions, beginning with detailed case descriptions, followed by thematic analysis and synthesis of lessons learned.

\section{Results of Phase 1: Common Elements and Key Points of Differentiation Across Studies}

In this section, we articulate each case's details, background, and context related to: (a) equipment, participants, and virtual 


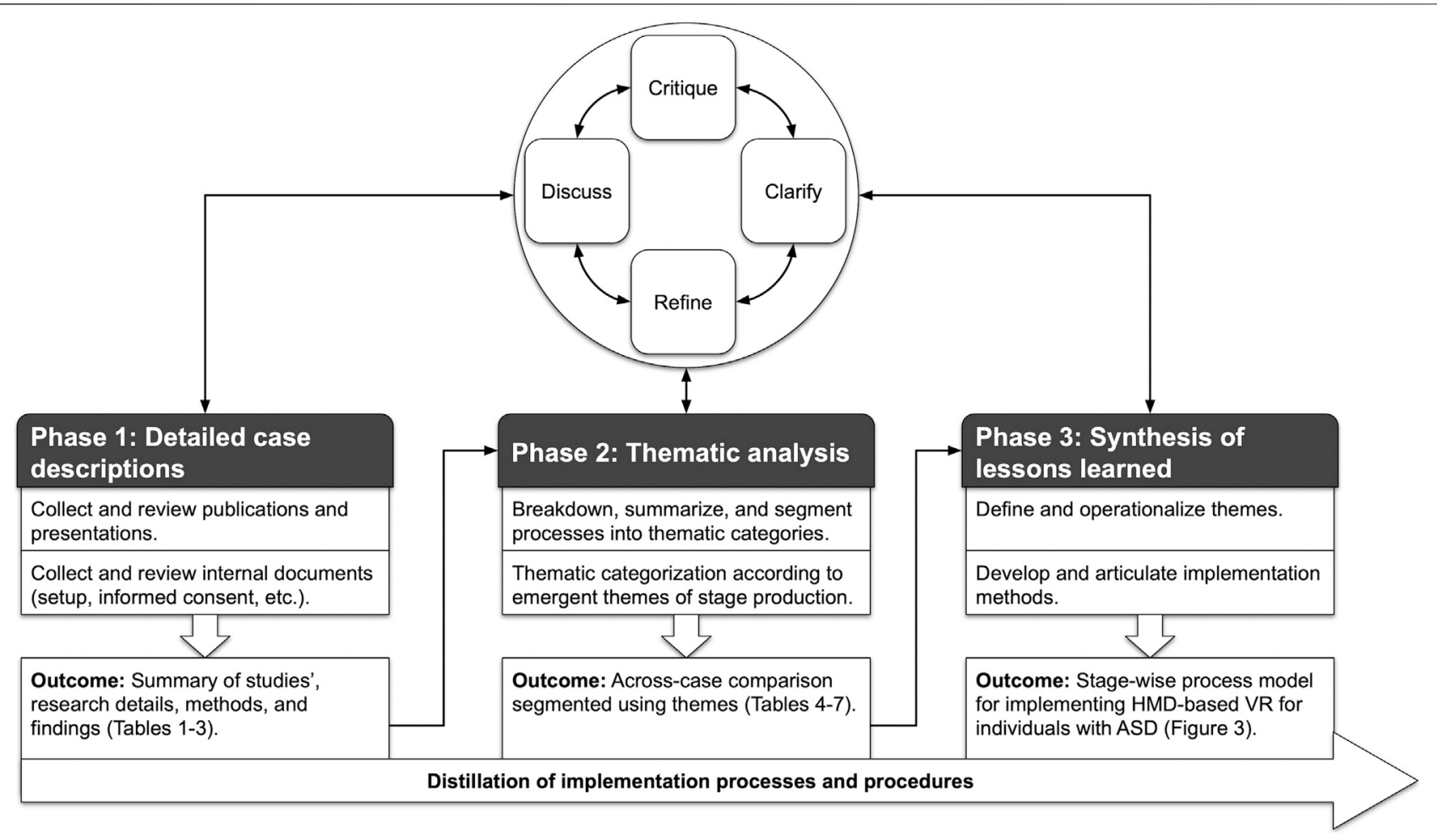

FIGURE 1 | Three-phase structured-iterative-comparative methodology used in this research.

TABLE 1 | Outcomes of Phase 1: Results from analysis of studies (equipment, participants, and virtual environments).

\section{Study 1}

\begin{tabular}{ll}
\hline Participants & Expert Testers (neurotypical) \\
& O 3 PhD-level experts; one staff member \\
O Purposive sample \\
ASD Participant Testers \\
O $N=5$ (all male, all verbal) \\
O Confirmed ASD diagnosis \\
O Age range = 23 to 34 \\
Convenience sample \\
O Lower tech \\
O Google Cardboard \\
O Google Daydream View \\
Higher tech \\
O HTC Vive \\
Birtual \\
Bespoke, custom-developed software \\
environment
\end{tabular}

\section{Study 2}

$N=31($ male $=25 ;$ female $=6)$

- Confirmed ASD diagnosis

- Verbal = 28; non-verbal $=3$

- Age range $=6$ to 16

Convenience sample

Lower tech

o Google cardboard

O ClassVR ${ }^{a}$

- Higher tech

- HTC Vive

- Off-the-shelf software

- Historically-focused environments and landscapes (i.e., Egypt)

Socially-focused environments (i.e., Fun Fair)

- Simulation experience (i.e., making a coffee in a coffee shop)

- All experiences single-user

${ }^{a}$ ClassVR is a low-cost, stand-alone VR headset designed specifically for fleet deployment in educational contexts.

environments (Table 1); (b) research aims, data collection protocols (Table 2); and (c) key findings and limitations (Table 3). Study 1 study utilized a design-based research (DBR) methodological approach that considered ease-of-use, the nature of participants' user experience, feasibility, and relevance of a spherical, video-based virtual reality (SVVR) app as well as a fully immersive, collaborative $3 \mathrm{D}$ virtual environment, both of which were delivered using HMDs for a 
TABLE 2 | Outcomes of Phase 1: Results from analysis of studies (research aims, data collection protocols).

United States study

United Kingdom study

Ethical review and

informed consent

Purpose and research questions

Data collection procedures

Data collected

\begin{abstract}
- Research approved by the Universities' Eth
- Parent and/or participant consent obtained

- Participant assent obtained as/where required

- To what extent do project prototypes meet design goals of being acceptable, feasible, easy to use, and relevant to the unique needs of participants?

- What is the nature of participants' user experience relative to the lower-tech and higher-tech simulations?
\end{abstract}

- Expert Testers (Spring, 2018)

O Experienced public transportation training simulation in two low tech HMDs for a period of up to $15 \mathrm{~min}$ (each) across two sessions at their workplace

O Sessions facilitated by trained graduate student

O Experts completed post-experience survey(s) and structured interviews

- Participant Testers (Summer, 2018)

O Experienced public transportation training simulation with one low tech device and one high tech device for a period of up to $15 \mathrm{~min}$ (each) at the offices of the adult day program

O Sessions facilitated by lead researcher and trained graduate student O Participants completed post-experience survey(s) and unstructured interviews

O An adult day program staff member who knew the participants well helped facilitate this

- Expert testers

O Semi-structured interview

O Self-report questionnaire (designed by research team)

- Participant testers

O Screen, webcam, and audio recordings

O Unstructured, post-usage testing interviews

O Qualitative field notes

- Both

O System usability scale (SUS)

O Adjectival user-friendliness scale
What type of VR HMD device and experiences therein are preferred by children on the autism spectrum?

- How do children on the autism spectrum report the physical experience, enjoyment, and potential of VR HMDs in their classrooms?

- What would children on the autism spectrum like to use VR for in schools?

- Participants tested all three HMDs for a period of up to 20 min (each) across two sessions in their school (Spring 2018)

- Sessions facilitated by lead researcher and teacher who knew the pupil well

- Participants exposed to virtual environments via Google Cardboard,

Class VR, and HTC Vive (in that order)

- Participants and teachers completed post-experience survey(s)

- Teachers helped to facilitate this for younger pupils and pupils with learning difficulties
Self-report questionnaires (designed by research team); completed by both ASD and teacher cohorts

- Qualitative field notes

- Focus group protoco

TABLE 3 | Outcomes of Phase 1: Results from analysis of studies (key findings and limitations).

Study 1

Key Above-average usability and good user-friendliness

findings

- All users were able to comp returned for additional session

- All participants indicated desire to use again

- Sense of enjoyment (i.e., joy, fun, excitement)

- Some evidence of adverse effects (i.e., nausea, dizziness)

Limitations

- Small sample

- Exploratory nature of the research

- Virtual environments were functional prototypes, not finished products

- Findings contextualized within adult day program and associated participants

\section{Study 2}

- A preference for using higher tech. A clear preference towards using/deploying VR HMDs for preparing for real life activities and visiting new places (vs. socialising and making friends)

- Preference for using VR to help calm and relax was a key opportunity for participants and teachers

- All participants indicated desire to use again

- Sense of enjoyment and ease of use

No adverse effects

- Small sample

- Exploratory nature of the research

- Exploration of VR software constrained by time

- Findings contextualized within UK schools included in the study and children in those schools group of adults with ASD in a day program. Study 2 sought to place children on the autism spectrum at the center of a study examining the potential of VR HMDs used in primary and secondary school classrooms; explored a range of VR experiences, how autistic users reported physical experiences, enjoyment, and potential of VR HMDs in their classrooms, while systematically exploring potential adverse effects. Detailed case descriptions are presented in Table 1. Cases are presented in a side-by-side tabular format to align each study's research details, methods, findings, etc. for thematic analysis in Phase 2. 
TABLE 4 | Results from thematic analysis: Stage 1 setting the stage.

Stage 1-Setting the stage

Processes

Common elements

Key points of

differentiation

United States

Needs assessment, stakeholder input/

meetings, design \& development of

intervention, IRB approval, expert testing

United Kingdom

Ethics approval, stakeholder meetings, formation

of RQs, school visits, show and tell with parents/

carers, practical issues related to tech in

classrooms explored, research mentor meetings

with lead researcher.
IRB/ethics approval; needs assessment, stakeholder input/meetings; expert testing (teachers, program managers, collaborators, etc. ..); designing/revising RQs with stakeholders; design/development of intervention and/or technology integration procedures.

TABLE 5 | Results from thematic analysis: Stage 2 Dress Rehearsal.

Stage 2-Dress rehearsal

Processes

Common elements

Key points of

differentiation

United States

Internal usage testing; hardware/software stability testing, locating ideal room; rehearsing procedures; developing setup/breakdown checklist; iteratively refining intervention protocol.
United Kingdom

Testing equipment in situ; locating ideal room; hardware/software stability testing; demo with teachers and parents; develop set-up checklist; final consultation; finalize protocol.
Testing equipment (hardware/software) in contexts/conditions of actual usage; including facilitators (teachers, staff, etc.); testing and refining protocols.

TABLE 6 | Results from thematic analysis: Stage 3 First Preview.

Stage 3-First preview

Processes

Common elements

Key points of

differentiation

United States

Present equipment to participants (see and touch devices), explore if participants are interested in using equipment, address any questions or concerns, preview the content, ask if participants want to participate, gain consent
United Kingdom

Present equipment to participants (a chance to see and touch devices), discuss any worries/ issues, watch video materials related to others using VR HMDs, preview and consider the content to be presented to participants, discuss any further/final worries concerns, gain consent from parents and pupils
Presentation of equipment and stimuli (in nonVR, screen-based format), continually address questions and queries from participants, complete consent process
Adult vs. child consent/ assent procedures

\section{Results of Phase 2: Emergent Themes from Comparison of Studies}

On the basis of the detailed case descriptions from Phase 1 (Tables 1-3), Phase 2 sought to present themes within and across cases. Results are framed within themes that emerged as lead researchers compared and analyzed cases. Over the process of thematic analysis, the researchers identified common segmentation points shared between cases. Seeking to sufficiently capture the complexity and highly contextual nature of our cases, the metaphor of stage production emerged. Stage production is particularly apt for characterizing our approach, as it seeks to gradually lead to a refined and reliably consistent performance that involves a range of stakeholders working together. As such, we characterize our implementations' segmentation points as "stages", and articulate these as: (a) setting the stage (Table 4); (b) dress rehearsal (Table 5); (c) first preview (Table 6); and (d) opening night (acts 1 and 2; Table 7). Within each stage, we briefly detail the processes underwent by each research team and highlight common elements and key points of differentiation. In line with our instrumental, collective case study methodology, the approach of structuring processes across cases in a stage-wise manner (see Tables 4-7) established the foundation for further interrogation and analytic generalization in Phase 3.

\section{Results of Phase 3: Lessons from Within- and Across-Case Comparison Related to Minimizing Adverse Effects of HMD-Based VR for Individuals with ASD}

Results presented in this section represent the final outcome of the 3-phase distillation procedure. Having identified points of 
TABLE 7 | Results from thematic analysis: Stage 4 Opening Night (Acts 1 and 2).

Stage 4-Opening Night: Act 1

\begin{tabular}{|c|c|c|c|}
\hline Processes & & Common elements & Key points of differentiation \\
\hline $\begin{array}{l}\text { United States } \\
\text { Ensure participant is sitting in swivel chair, } \\
\text { explain the task structure while showing } \\
\text { how to navigate the app, ask if participant } \\
\text { is comfortable and ready to begin, fit the } \\
\text { headset (Cardboard or Daydream), ask if } \\
\text { participant is comfortable and willing to } \\
\text { continue, ask participant to complete first } \\
\text { task ( } 2 \text { min), provide assistance if needed, } \\
\text { ask how participant is feeling and if } \\
\text { comfortable and ready to begin next task, } \\
\text { repeat after each task }(n=4) \text { until activity is } \\
\text { completed }\end{array}$ & $\begin{array}{l}\text { United Kingdom } \\
\text { Explain how the session will run, sit } \\
\text { participant down, introduce first HMD } \\
\text { experience using low tech device } \\
\text { (Cardboard), check they still want to } \\
\text { proceed, fit the HMD, run application ( } 2-3 \\
\text { min) then remove HMD, check for negative } \\
\text { effects (ask participant and observe), run } \\
\text { further HMD experience, complete activity } \\
\text { ( }<5 \text { min), check for negative effects, follow up } \\
30 \text { min later for signs of negative effects }\end{array}$ & $\begin{array}{l}\text { Deliberate orientation and training } \\
\text { process; ensure participant is sitting; initial } \\
\text { trial; continually check participants } \\
\text { willingness to use at set intervals; } \\
\text { continually check for adverse effects; } \\
\text { short lengths of exposure }\end{array}$ & $\begin{array}{l}\text { Follow up on negative effects } \\
\text { beyond the formalities of the study }\end{array}$ \\
\hline
\end{tabular}

Stage 4-Opening Night: Act 2

Processes

United States

Ensure participant is sitting in swivel chair, show HTC Vive controllers, show how to hold controllers, ask participant to press different buttons on controllers, ask participant if they are comfortable and ready to try on the $\mathrm{HTC}$ Vive $\mathrm{HMD}$, fit the $\mathrm{HMD}$, ask participant if it is comfortable and they are willing to continue, have participant perform simple navigation task (find their cubicle in the office; 1-2 min), provide assistance if needed, ask participant if task was easy or difficult, ask how participant is feeling, ask if participant is comfortable and willing to continue, engage in full activity ( 9 min), check for negative effects
United Kingdom

Explain how the session will run, researcher uses HTC Vive to demonstrate activity (and controls), sit/stand participant (depending on their age), check they still want to proceed, fit the HMD and hand controls, ensure comfort, run application (2-3 min) then remove HMD, follow the participant around the space/room (if standing/walking) to ensure stable balance, check for negative effects (ask participant and observe), run further HMD experience, complete activity ( $<5 \mathrm{~min}$ ), check for negative effects, follow up 30 min later for signs of negative effects

\section{Common elements}

Deliberate orientation and training process; initial trial; continually check participants willingness to use at set intervals; continually check for adverse effects; provide assistance

\section{Key points of differentiation}

Length of exposure; standing vs. sitting; multi-user vs. single-user virtual environment convergence and divergence across cases in phase 2, our focus transitioned in phase 3 to integrating the processes from both studies (Tables 4-7). Considerations of how to convey the lessons learned from prior analysis presented an opportunity to merge the processes and common elements from both cases into a representation of best practices. This required a translation of the specific details of each case into a set of generalizations that described each of the implementation stages. Attempts to develop textual descriptions of phases led to tensions, as text-based descriptions did not sufficiently represent the linear, step-wise nature of the implementation processes uncovered in our analysis. This led us to develop an initial linear visualization of our processes, which we subsequently refined using the approach shown in Figure 1 above. As a result, we articulated the stage-wise implementation framework as a linear process with descriptions and intent of each stage. Resonant in this process was the concept of gradual acclimation, which we discuss as follows.

\section{Gradual Acclimation for Minimizing Adverse Effects} To minimize potential adverse effects gradual acclimation is needed (Figure 2). This is the process through which VR is continually optimized within and across implementation stages. We articulate gradual acclimation in two different areas of the process model: (1) contextual acclimation (Stages 1 and 2) and (2) technology acclimation (Stages 3 and 4). Contextual acclimation is concerned with researchers identifying the local variables that potentially could impact successful implementation. Contextual acclimation is primarily informed via input from stakeholders and facilitators (e.g., teachers, staff, program managers, collaborators, directors). Contextual acclimation is followed by technology acclimation, which is informed principally by participants when they begin by handling and experiencing the technology in a gradual manner in stages 3 and 4 .

Gradual acclimation is, in part, predicated on ethical concerns. When working with a vulnerable population, researchers have a special obligation and greater responsibility to actively take precautions to help minimize real or potential risks (American Educational Research Association, 2011; Behavior Analyst Certification Board, 2014). This extends to possible adverse effects associated with the use of HMD-based VR for individuals ASD. We therefore assert that the potential for adverse effects could be minimized through intentional procedures that promote gradual acclimation within and 


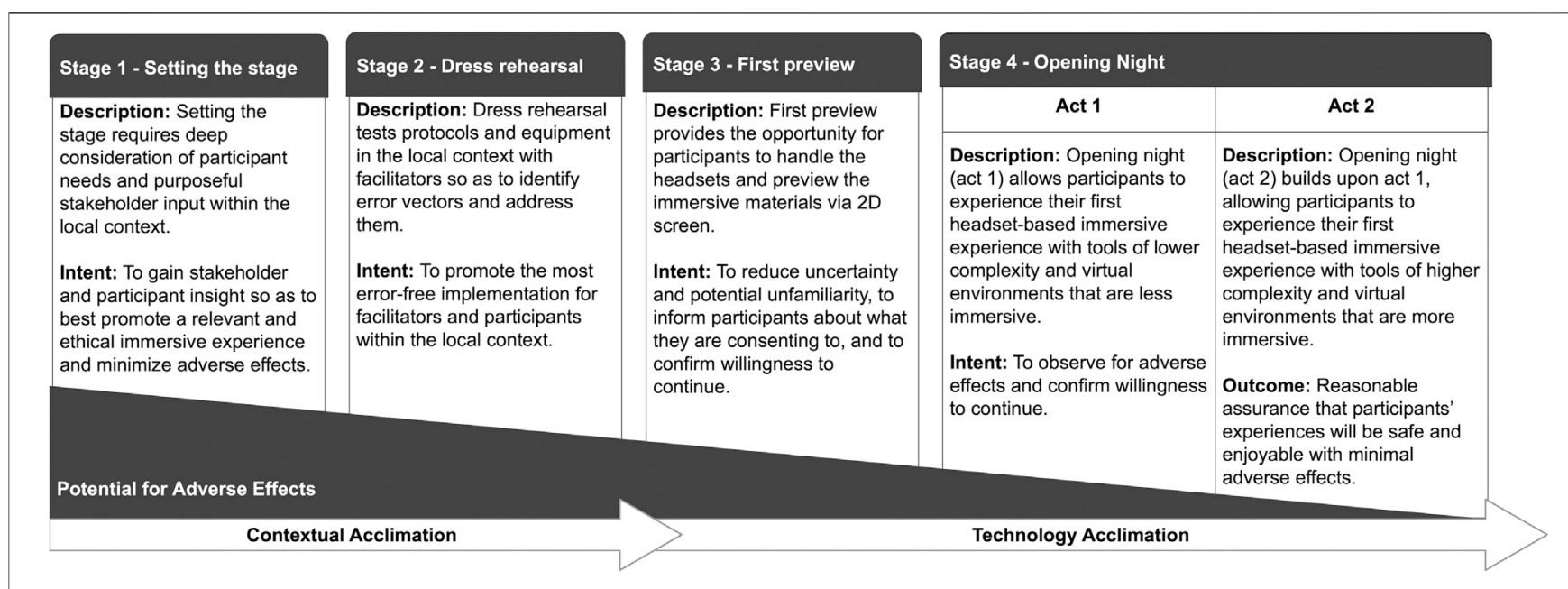

FIGURE 2 | Final stage-wise process model promoting gradual acclimation to minimize potential adverse effects of HMD-based VR for individuals with ASD.

across all stages. This is captured in Figure $\mathbf{2}$ as a triangle labeled Potential for adverse effects, which illustrates how the potential for adverse effects could be reduced by utilizing the intentional and systematic processes through contextual acclimation and technology acclimation. Figure 2 represents our final stagewise process model of gradual contextual and technology acclimation to minimize potential adverse effects of HMDbased VR for individuals with ASD.

\section{DISCUSSION}

Auspicious claims championing the potential of VR for individuals with ASD are abundant in the literature of the past 25 years-from the earliest reports (e.g., Strickland, 1996; 1997) to the most recent (Miller et al., 2019; Ke et al., 2020). However, "While it is apparent that VR has great conceptual potential, research and development are early on the road to making it a reality" (Wang and Anagnostou, 2014, p. 2137). Contributing to this are the many gaps in this research area, including a lack of guidelines for implementing VR interventions with this population, a general lack of consideration of adverse effects of VR in the published research, and very little specific research that reports findings related to adverse effects. Given the known characteristics of people with ASD (Christensen et al., 2018), and compounded by common comorbidities (Deprey and Ozonoff, 2018) such as anxiety (van Steensel and Heeman, 2017) and sensory processing differences (Thye et al., 2018), the prospect of adverse effects related to VR usage for individuals with ASD presents salient risks. Approaching calls and concerns for the safe and ethical applied use of technology for individuals with autism (Parsons, 2016; Bradley and Newbutt, 2018; Schmidt et al., 2019), the current article approaches the urgent need to consider potential adverse effects of HMD-based VR use for individuals with ASD.

To confront this need, we present a stage-wise process model derived from our own research that illustrates how researchers and practitioners alike can seek to reduce potential adverse effects in the design and execution of their HMD-based VR implementations for individuals with ASD. To contextualize the embedded processes and promote recall, we adopt the metaphor of a stage production to describe the different stages of the process. Like stage production, VR implementation requires considerable attention to details, many of which are unknown until they are encountered. Successful VR implementation also requires intentional, continuous coordination with key stakeholders such as teachers, administrators, IT staff, etc. (Newbutt, 2019), similar to a stage production requiring coordination between theater and production staff (e.g., stage manager, stagehands). Further, and perhaps most importantly, planning opportunities for participants to encounter VR technology and practice using it before engaging in an actual intervention is as important to VR implementation as is memorization of lines and tablereads to actors' performance in a stage production. Finally, in stage production, preparation and rehearsal are critically important to bringing the production to a high standard of quality. We posit that application of the stage-wise model presented here could likewise influence the quality of the VR experience for participants by controlling for potential adverse effects.

\section{Stage-Wise Process Model}

The stage-wise process model represents a distillation of how two separate research teams approached the challenge of controlling for potential adverse effects. Our intent here is to provide guidance in an approachable, actionable framework and in doing so to portray our model in a minimalist manner so as to be useful to a range of audiences. However, this introduces a risk that it could be interpreted as an oversimplification of what in many cases can be exceptionally complex processes. The reader is therefore cautioned not to interpret the model at face value, but instead to consult the specific information presented in Tables 4-7 for detailed examples of the specific procedures and activities that were applied by using each stage of our work. We provide further discussion of the stages in the following sections. 


\section{Stage 1: Setting the Stage}

Stage 1 includes front-end analysis and subsequent design and development of the intervention. These are activities that are well documented in the learning and instructional design literature (Morrison et al., 2012; Dick et al., 2014). What differentiates our approach from such established instructional design processes, however, is intentional solicitation of collaborative and meaningful input from all stakeholders, beginning in Stage 1, and continuing across all stages of the process. Continual input from stakeholders ensures the voices of those who matter the most are heard (Parsons et al., 2020). We highlight that this goes beyond requirements of institutional review boards or ethics committees for human subjects research (Protection of Human Subjects, 2009). Catalyzing this were critiques rebuking research studies that: (1) do not incorporate the voices and perspectives of individuals with ASD, their support communities, and/or relevant stakeholders in the design, implementation, and/or execution of studies; and (2) do not pursue and incorporate the priorities and cultural values of autistic communities (Parsons, 2016; Parsons et al., 2020). Importantly, teams of researchers and stakeholders should explicitly acknowledge in this stage that VR use and/or intervention has inherent potential for adverse effects. As such, they should actively seek to identify factors that could promote reduction of adverse effects as early as this stage. For example, a team might discover that some participants find the fit of one headset to be disagreeable, which could prompt exploration of more adjustable straps or different headsets altogether.

\section{Stage 2: Dress Rehearsal}

In Stage 2, the VR technology used to deliver the intervention is tested in-situ. This testing allows teams to collectively examine the space where the technology will be used. When working in$s i t u$, researchers could find themselves provided with a space that is not ideal for HMD-based VR application. For example, they could be assigned a room that is too small, a cluttered area, or spaces containing tables and chairs. Such a space can present collision and trip hazards, which could lead to adverse health effects. Further, space needs to be amenable to a positive sensory experience for participants. For example, fluorescent lights or loud noises could be severely distracting for individuals with ASD (Grandin, 2002). In addition to this, testing allows the team to set up, configure and test the technology outside of a controlled lab setting. This allows for discovery of a variety of potential error points, for example, connectivity challenges such as restricted networks and prohibitive firewall rules, logistical challenges such as setting up for multi user experiences, or computer problems, such as misconfigurations and unforeseen glitches. Importantly, dress rehearsal is not technology testing; it is preparing for socialbehavioral human subjects research (American Psychiatric Association, 2013). As such, it provides opportunities for stakeholders to see and experience the application of the technology and provide feedback, with the ultimate goal of providing an error-free, enjoyable implementation by identifying and addressing vectors that potentially could lead to adverse effects such as confusion, frustration, etc.

\section{Stage 3: First Preview}

In Stage 3, focus shifts to technology usage by participants. In line with the principle of gradual acclimation, this stage provides participants the opportunity to examine the technology and learn about the intervention. Many individuals with ASD respond positively to order, structure, and predictability, with deviations potentially leading to increased stress and anxiety (Gotham et al., 2013; Lidstone et al., 2014; Factor et al., 2016). First Preview seeks to address this by providing structured opportunities to communicate the project to participants. Although research studies are often communicated to research participants through approved informed consent procedures (e.g., American Educational Research Association, 2011; British Educational Research Association, 2018), we have found in our work the language used to describe VR projects (as approved by institutional review boards or ethical committees) can be abstract and contextually dependent. Given that many individuals with ASD are quite literal in their thinking (Volden et al., 2009; Deliens et al., 2018), this can frustrate communication efforts. The purpose of providing the opportunity for examining the technology and previewing the VR materials on a monitor is to provide concrete and real examples of what they will be experiencing, thereby enhancing communication with our participants. In this manner, two key aspects are achieved: (1) we are ensuring our ethical obligation to our participants (i.e., clear and concise communication); and (2) vulnerable participants are able to make betterinformed decisions about whether they choose to participate in a given research study and what to expect if they do agree to be involved. This again illustrates the importance of privileging the voices and perspectives of participants throughout the implementation process (Parsons and Cobb, 2013; Politis et al., 2019).

\section{Stage 4: Opening Night Act 1}

In Act 1 of Opening Night the equipment and experience is delivered in a limited manner so as to promote gradual acclimation. Examples include: limiting the amount of time using the equipment; using lower tech versions of the equipment; and/or using simplified versions of the equipment. First, limiting the amount of time that participants engage in the VR experience could reduce the probability of serious adverse effects and allows researchers to check frequently with participants regarding how they are feeling, as well as with their guardians, caregivers, educators, etc. For example, researchers might design a series of 2-min tutorials or short 360 videos and take breaks in between to evaluate participants' well-being and willingness to proceed. Second, using lower tech versions of the technology could be less complicated and therefore provide superior usability (Parish-Morris et al., 2018; Schmidt et al., 2019). For example, Google Cardboard devices have only a single button and no straps, allowing for the HMD to be removed easily in the case that a participant is experiencing negative effects. Third and finally, using simplified versions of the equipment, software, etc. could provide authentic experiences while reducing potential frustration or confusion (Rojo et al., 
2019). For example, a series of 360 videos might auto-play instead of requiring participants to operate controls, or short tutorials might be presented using an "on rails" design in which participants are limited to moving their avatar, making selections, or changing their view, but not controlling the path their avatar takes. Given that the local context necessarily circumscribes how this stage of implementation is designed, these examples are not meant to be prescriptive but instead to illustrate how gradual acclimation is realized in this stage. Importantly, researchers must take care to intentionally and often question participants and other advocates who are involved regarding their well-being. Given recognized communication differences in many individuals with ASD (Masi et al., 2017), soliciting responses is recommended over providing opportunities for self-initiated reporting. This requires careful discussion and agreement to be sure that, for example, non-verbal participants can express their willingness to continue or desire to end the experience and/or study.

\section{Stage 4: Opening Night Act 2}

Act 2 of Opening Night is when participants engage comprehensively in the VR experience using fully immersive headsets (i.e., Oculus Quest, HTC Vive). During this stage, researchers should remain vigilant in their observation of participants while they engage in the experience(s), scrutinizing for any signs of adverse effects. Assuming that the prior stages have been enacted in a considered manner, participants should have gradually acclimatized to the technology, and the likelihood that adverse effects would unfavorably impact participants in this stage should be diminished.

Efforts to minimize risk do not end at this point. Even though a VR intervention might be very feasible for certain applications initially, using the VR intervention might become frustrating over time or introduce new risks. Evidence of this is scant given the lack of longitudinal research in this area. However, emerging research suggests longitudinal exposure effects of VR with individuals with ASD (Glaser et al., 2020), with participants rating their perceptions of cybersickness higher in later sessions and as the VR environment increased in complexity. Therefore, after participants have begun a training program or intervention, vigilance in observation should be ongoing and continual adjustments should be made as needed.

Through the stages presented above and the synthesised data from a US and a UK-based study, we have addressed an established lack of explicit guidance (Newbutt, 2019). On the one hand, research has noted the potential for VR HMDs to induce some negative effects (Sharples et al., 2008; Chessa et al., 2019; Weech et al., 2019), while on the other, very limited advice is offered on how to practically overcome these challenges. This is especially true in the field of autism, in which we are working with individuals who can present with sensory concerns (American Psychiatric Association, 2013). Our results provide initial insights to both fill a gap in the literature and also promote safe HMDbased VR practices for individuals with ASD and their support communities.

\section{Implications}

In the current article, we have presented a stage-wise process model promoting gradual acclimation to minimize potential adverse effects of HMD-based VR for individuals with ASD. We do not assert that these procedures are novel of-themselves. Instead, the innovation of our approach rests in how we distilled specific processes from successful research studies which could be used by others potentially to guide their own implementations. Further, our process model intentionally embraces and embodies social disability models, foregrounding themes of dignity, agency, and empowerment (Braddock et al., 2013). By locating the perspectives and voices of individuals with ASD centrally, we seek to avoid the mistake of insufficiently considering neurophilosophical issues such as hyper- or hyporeactivity to sensory inputs that individuals with ASD may experience (Tavassoli et al., 2016; Uljarević et al., 2016). While the impetus for developing the stage-wise process model was to provide guidance for reducing potential adverse effects, benefits of using the process model could represent a generalizable set of guidelines that others could use to inform their VR implementations. Inclusion of stakeholders and participants across all stages of implementation could increase their confidence in potential benefits of using the technology, positively influence potential anxiety on the part of participants, and increase stakeholder confidence that the technology will not cause harm. Designers could map existing methods and processes to the various stages in the model and use this to inform and guide their designs. Ultimately, the process model we provide in Figure 2 and discuss above could provide some assurance that stakeholders are being supportive of their ethical responsibilities when working with a vulnerable population (Bell, 2008; Pittaway et al., 2010).

\section{Limitations}

The research presented in the current article represents an important first step in reporting how researchers approached the problem of potential adverse effects in their implementation procedures. However, researchers seeking to utilize and further develop our approach should be aware of its limitations. The purpose of the research presented here was not to validate the stage-wise process model. While we believe it is likely that following a stage-wise implementation process helped to minimize adverse effects, we do not currently have sufficient data to support a causal relationship between the stage-wise process model and reduction in adverse effects. Because this work drew from completed studies, it was not possible to establish whether our stage-wise implementation processes were specifically related to the general lack of adverse effects observed in both studies. In addition, despite our collaborative approach working with individuals with ASD, we have not yet returned to these communities with our final process model to solicit feedback and input-a direction for future research. Furthermore, to extend the work presented here and approach limitations identified above, future research should explore the empirical relationship between utilization of our process model and influence on adverse effects. Moreover, given the heterogenous nature of ASD, future research should address 
how to tailor the process model in consideration of factors such as necessary level of support, individual differences (i.e., reactivity to sensory inputs, levels of cognition, etc.), and extended exposure to HMD technologies.

\section{CONCLUSION}

The focus in the current article on adverse effects of HMD-based VR for individuals with ASD rests on the basis of ethical guidelines published in our respective research contexts, including but not limited to those of BERA (British Educational Research Association, 2018), AERA (American Educational Research Association, 2011), and BACB (Behavior Analyst Certification Board, 2014). We situate the respect and dignity of our participants at the forefront of our work and incorporate practices that promote inclusion and involvement of participants and stakeholders. Paramount to our work is an acute sensitivity to the vulnerability of our target population (Parsons, 2015). In light of this, we endeavor to honor our special obligation and the greater responsibility we have as researchers to actively take precautions to help minimize real or potential risks associated with the use of HMD-based VR for ASD (e.g.,

\section{REFERENCES}

American Educational Research Association. (2011). Code of ethics. Available at: https://www.aera.net/Portals/38/docs/About_AERA/CodeOfEthics(1).pdf

American Psychiatric Association. (2013). Diagnostic and statistical manual of mental disorders. Washington, DC: American Psychiatric Association.

Bord, K. (2007). Wicked ID: conceptual framework for considering instructional design as a wicked problem. Cjlt/Rcat 33 (1), n1. doi:10.21432/t2cg6h

Behavior Analyst Certification Board (2014). Professional and ethical compliance code for behavior analysts. Available at: https:/www.bacb.com/wp-content/ uploads/2020/05/BACB-Compliance-Code-english_190318.pdf

Bell, N. (2008). Ethics in child research: rights, reason and responsibilities. Child. Geogr. 6 (1), 7-20. doi:10.1080/14733280701791827

Bellani, M., Fornasari, L., Chittaro, L., and Brambilla, P. (2011). Virtual reality in autism: state of the art. Epidemiol. Psychiatr. Sci. 20, 235-238. doi:10.1017/ s2045796011000448

Benton, L., Ashwin, E., Johnson, H., Grawemeyer, B., and Brosnan, M. (2011). IDEAS: An interface design experience for the autistic spectrum, 1759-1764. Berlin: Springer.

Bozgeyikli, L., Raij, A., Katkoori, S., and Alqasemi, R. (2018). A survey on virtual reality for individuals with autism spectrum disorder: design considerations. IEEE Trans. Learn. Technol. 11 (2), 133-151. doi:10.1109/tlt.2017.2739747

Braddock, D., Hoehl, J., Tanis, S., Ablowitz, E., and Haffer, L. (2013). The rights of people with cognitive disabilities to technology and information access. Inclusion 1 (2), 95-102. doi:10.1352/2326-6988-01.02.95

Bradley, R., and Newbutt, N. (2018). Autism and virtual reality head-mounted displays: a state of the art systematic review. J. Enabling Tech. 12, 101-113. doi:10.1108/jet-01-2018-0004

British Educational Research Association. (2018). Ethical guidelines for educational research. Available at: https://www.bera.ac.uk/researchers-resources/ publications/ethicalguidelines-for-educational-research-2018

Chessa, M., Maiello, G., Borsari, A., and Bex, P. J. (2019). The perceptual quality of the oculus rift for immersive virtual reality. Human-Comput. Interact. 34 (1), 51-82. doi:10.1080/07370024.2016.1243478

Christensen, D. L., Braun, K. V. N., Baio, J., Bilder, D., Charles, J., Constantino, J. N., et al. (2018). Prevalence and characteristics of autism spectrum disorder among children aged 8 Years - autism and developmental disabilities emotional and physical harm or intervention side-effects). These potential risks could represent environmental constraints that we as researchers have an ethical obligation to eliminate. To this end, we have developed methods and processes for minimizing adverse effects of HMD-based VR for individuals with ASD. Our sincere hope is that the work presented here can serve as a signpost for how researchers can ethically approach considerations of adverse effects in future HMD-based VR implementations, and we urge researchers to consider applying and empirically interrogating the model proposed here.

\section{DATA AVAILABILITY STATEMENT}

The original contributions presented in the study are included in the article, further inquiries can be directed to the corresponding author.

\section{AUTHOR CONTRIBUTIONS}

All authors listed have made a substantial, direct and intellectual contribution to the work, and approved it for publication.

monitoring network, 11 sites, United States, 2012. MMWR Surveill. Summ. 65 (13), 1. doi:10.15585/mmwr.ss6513a1

Deliens, G., Papastamou, F., Ruytenbeek, N., Geelhand, P., and Kissine, M. (2018). Selective pragmatic impairment in autism spectrum disorder: indirect requests versus irony. J. Autism Dev. Disord. 48 (9), 2938-2952. doi:10.1007/s10803-018-3561-6

Deprey, L., and Ozonoff, S. (2018). Assessment of comorbid psychiatric conditions in autism spectrum disorder. Berlin: Springer.

Dick, W., Carey, L., and Carey, J. (2014). The systematic design of instruction. 8th Edn. London: Pearson.

DSM-5 American Psychiatric Association. (2013). Diagnostic and statistical manual of mental disorders. New York, NY: American Psychiatric Publishing.

Eaves, L. C., and Ho, H. H. (2008). Young adult outcome of autism spectrum disorders. J. Autism Dev. Disord. 38, 739-747. doi:10.1007/s10803-007-0441-x

Estes, A., Munson, J., Rogers, S. J., Greenson, J., Winter, J., and Dawson, G. (2015). Long-term outcomes of early intervention in 6-year-Old children with autism spectrum disorder. J. Am. Acad. Child Adolesc. Psychiatry 54 (7), 580-587. doi:10.1016/j.jaac.2015.04.005

Factor, R. S., Condy, E. E., Farley, J. P., and Scarpa, A. (2016). Brief report: insistence on sameness, anxiety, and social motivation in children with autism spectrum disorder. J. Autism Dev. Disord. 46 (7), 2548-2554. doi:10.1007/ s10803-016-2781-x

Fletcher-Watson, S. (2014). A targeted review of computer-assisted learning for people with autism spectrum disorder: towards a consistent methodology. Rev. J. Autism Dev. Disord. 1 (2), 87-100. doi:10.1007/s40489-013-0003-4

Freina, L., and Ott, M. (2015). A literature review on immersive virtual reality in education: state of the art and perspectives. Virt. Real. 1, 133. doi:10.1007/ s10055-020-00489-9

Glaser, N. J., and Schmidt, M. (2018). Usage considerations of 3D collaborative virtual learning environments to promote development and transfer of knowledge and skills for individuals with autism. Tech. Know. Learn. 25 (25), 315-322. doi:10.1007/s10758-018-9369-9

Glaser, N., Schmidt, M., and Schmidt, C. (2020). Fear and loathing in VR: cybersickness evidence in headset-based VR training for adults with Autism Presented at the 2020 international convention of the association for educational communications and technology. Online.

Gotham, K., Bishop, S. L., Hus, V., Huerta, M., Lund, S., Buja, A., et al. (2013). Exploring the relationship between anxiety and insistence on sameness in autism spectrum disorders. Autism Res. 6 (1), 33-41. doi:10.1002/aur.1263 
Grandin, T. (2002). Teaching tips for children and adults with autism, 2. Fort Collins: Colorado/EUA, 5 .

Grynszpan, O., Weiss, P. L., Perez-Diaz, F., and Gal, E. (2014). Innovative technology-based interventions for autism spectrum disorders: a metaanalysis. Autism 18 (4), 346-361. doi:10.1177/1362361313476767

Hedley, D., Uljarević, M., Cameron, L., Halder, S., Richdale, A., and Dissanayake, C. (2017). Employment programmes and interventions targeting adults with autism spectrum disorder: a systematic review of the literature. Autism 21 (8), 929-941. doi:10.1177/1362361316661855

Irish, J. E. N. (2013). Can I sit here? A review of the literature supporting the use of single-user virtual environments to help adolescents with autism learn appropriate social communication skills. Comput. Hum. Behav. 29 (5), A17-A24. doi:10.1016/j.chb.2012.12.031

Jarrold, W., Mundy, P., Gwaltney, M., Bailenson, J., Hatt, N., McIntyre, N., et al. (2013). Attention in a virtual public speaking task in higher functioning children with autism. Autism Res. 6, 393-410. doi:10.1002/aur.1302

Josman, N., Ben-Chaim, H. M., and Friedrich, S. (2011). Effectiveness of virtual reality for teaching street-crossing skills to children and adolescents with autism. Int. J. Disabil. Hum. Dev. IJDHD 7 (1), 49-56. doi:10.1515/ijdhd. 2008.7.1.49

Kandalaft, M. R., Didehbani, N., Krawczyk, D. C., Allen, T. T., and Chapman, S. B. (2013). Virtual reality social cognition training for young adults with highfunctioning autism. J. Autism Dev. Disord. 43 (1), 34-44. doi:10.1007/s10803012-1544-6

Ke, F., and Im, T. (2013). Virtual-reality-based social interaction training for children with high-functioning autism. J. Educ. Res. 106 (6), 441-461. doi:10. 1080/00220671.2013.832999

Ke, F., Moon, J., and Sokolikj, Z. (2020). Virtual reality-based social skills training for children with autism spectrum disorder. J. Spec. Education Technol. 14, 016264342094560. doi:10.1109/fie44824.2020.9273818

Kellmeyer, P. (2018). Neurophilosophical and ethical aspects of virtual reality Therapy in Neurology and Psychiatry. Camb Q. Healthc. Ethics 27 (4), 610-627. doi:10.1017/s0963180118000129

Khowaja, K., and Salim, S. S. (2013). A systematic review of strategies and computer-based intervention (CBI) for reading comprehension of children with autism. Res. Autism Spectr. Disord. 7 (9), 1111-1121. doi:10.1016/j.rasd. 2013.05.009

Lidstone, J., Uljarević, M., Sullivan, J., Rodgers, J., McConachie, H., Freeston, M., et al. (2014). Relations among restricted and repetitive behaviors, anxiety and sensory features in children with autism spectrum disorders. Res. Autism Spectr. Disord. 8 (2), 82-92. doi:10.1016/j.rasd.2013.10.001

Lincon, Y. S., and Guba, E. G. (1985). Naturalistic inquiry. Sage: Beverly Hills.

Malihi, M., Nguyen, J., Cardy, R. E., Eldon, S., Petta, C., and Kushki, A. (2020). Short report: evaluating the safety and usability of head-mounted virtual reality compared to monitor-displayed video for children with autism spectrum disorder. Autism 24, 1924-1929. doi:10.1177/1362361320934214

Masi, A., DeMayo, M. M., Glozier, N., and Guastella, A. J. (2017). An overview of autism spectrum disorder, heterogeneity and treatment Options. Neurosci. Bull. 33 (2), 183-193. doi:10.1007/s12264-017-0100-y

Mesa-Gresa, P., Gil-Gómez, H., Lozano-Quilis, J.-A., and Gil-Gómez, J.-A. (2018). Effectiveness of virtual reality for children and adolescents with autism spectrum disorder: an evidence-based systematic review. Sensors 18 (8), 114. doi: $10.3390 / s 18082486$

Miller, I. T., Wiederhold, B. K., Miller, C. S., and Wiederhold, M. D. (2019). Virtual reality air travel training with children on the autism spectrum: a preliminary report. Cyberpsychol. Behav. Soc. Netw. 23, 10-15. doi:10.1089/cyber.2019.0093

Mitchell, P., Parsons, S., and Leonard, A. (2007). Using virtual environments for teaching social understanding to 6 adolescents with autistic spectrum disorders. J. Autism Dev. Disord. 37 (3), 589-600. doi:10.1007/s10803-006-0189-8

Moore, D., Yufang Cheng, P., and McGrath, P. (2005). Collaborative virtual environment technology for people with autism. Focus Autism Other Dev. Disabl. 20, 231-243. doi:10.1177/10883576050200040501

Morrison, G. R., Ross, S., Kalman, H., and Kemp, J. (2012). Designing effective instruction. 7th Edn. Hoboken: John Wiley.

Newbutt, N., Sung, C., Kuo, H.-J., Leahy, M. J., Lin, C.-C., and Tong, B. (2016). Brief report: a Pilot study of the use of a virtual reality headset in autism populations. J. Autism Dev. Disord. 46 (9), 3166-3176. doi:10.1007/s10803-016$2830-5$
Newbutt, N., Sung, C., Kuo, H-J., and Leahy, M. J. (2017). "The potential of wearable technology (head-mounted displays) and virtual reality to support people with autism: acceptance, challenges, and future applications," in Recent advances in technologies for inclusive well-being: from worn to off-body sensing, virtual worlds, and games for serious applications. Editors A. Brooks, S. Brahnam, and L. Jain (Berlin: Springer), 221-241. Chapter 11.

Newbutt, N., Bradley, R., and Conley, I. (2020). Using virtual reality head-mounted display in schools for pupils on the autism spectrum; views, experiences and future directions. Cyberpsychol. Behav. Soc. Netw. 23 (1), 22-33. doi:10.1089/ cyber.2019.0206

Newbutt, N. (2019). "Assisting people with autism spectrum disorder through technology," in Encyclopedia of education and information technologies. Editor A. Tatnall (Switzerland: Springer).

Palmisano, S., Mursic, R., and Kim, J. (2017). Vection and cybersickness generated by head-and-display motion in the Oculus Rift. Displays 46, 1-8. doi:10.1016/j. displa.2016.11.001

Parish-Morris, J., Solórzano, R., Ravindran, V., Sazawal, V., Turnacioglu, S., Zitter, A., et al. (2018). Immersive virtual reality to improve police interaction skills in adolescents and adults with autism spectrum disorder: preliminary results of a phase i feasibility and safety trial. Annu. Rev. CyberTherapy Telemed. 14 (16), 50-56. doi:10.1002/aur.2352

Parsons, S., and Cobb, S. (2013). EPSRC observatory for responsible innovation in ICT. Who chooses what I need? Child voice and user-involvement in the development of learning technologies for children with autism. Berlin: Springer.

Parsons, S., Mitchell, P., and Leonard, A. (2004). The use and understanding of virtual environments by adolescents with autistic spectrum disorders. J. Autism Dev. Disord. 34 (4), 449-466. doi:10.1023/b:jadd.0000037421.98517.8d

Parsons, S., Yuill, N., Good, J., and Brosnan, M. (2020). 'Whose agenda? Who knows best? Whose voice?' Co-creating a technology research roadmap with autism stakeholders. Disabil. Soc. 35 (2), 201-234. doi:10.1080/09687599.2019.1624152

Parsons, S. (2015). Learning to work together: designing a multi-user virtual reality game for social collaboration and perspective-taking for children with autism. Int. J. Child-Comput. Interact. 6, 28-38. doi:10.1016/j.ijcci.2015.12.002

Parsons, S. (2016). Authenticity in virtual reality for assessment and intervention in autism: a conceptual review. Educ. Res. Rev. 19, 138-157. doi:10.1016/j.edurev. 2016.08.001

Pittaway, E., Bartolomei, L., and Hugman, R. (2010). 'Stop stealing our stories': the ethics of research with vulnerable groups. J. Hum. Rights Pract. 2 (2), 229-251. doi:10.1093/jhuman/huq004

Politis, Y., Olivia, L., and Olivia, T. (2019). Empowering autistic adults through their involvement in the development of a virtual world. Aia 5 (4), 303-317. doi:10.1108/aia-01-2019-0001

Protection of Human Subjects (2009). CFR 45, 46.

Rojo, D., Mayor, J., Rueda, J. J. G., and Raya, L. (2019). A virtual reality training application for adults with Asperger's syndrome. IEEE Comput. Grap. Appl. 39 (2), 104-111. doi:10.1109/mcg.2018.2884272

Potel, G., Rodgers, L. R., Ukoumunne, O. C., and Ford, T. (2014). Prevalence of parentreported ASD and ADHD in the UK: findings from the Millennium Cohort study. J. Autism Dev. Disord. 44 (1), 31-40. doi:10.1007/s10803-013-1849-0

Schmidt, M., Laffey, J. M., Schmidt, C. T., Wang, X., and Stichter, J. (2012). Developing methods for understanding social behavior in a 3D virtual learning environment. Comput. Hum. Behav. 28 (2), 405-413. doi:10.1016/j.chb.2011. 10.011

Schmidt, M., Schmidt, C., Glaser, N., Beck, D., Lim, M., and Palmer, H. (2019). Evaluation of a spherical video-based virtual reality intervention designed to teach adaptive skills for adults with autism: a preliminary report. Interact. Learn. Environ. 11, 33. doi:10.1080/10494820.2019.1579236

Self, T., Scudder, R. R., Weheba, G., and Crumrine, D. (2007). A virtual approach to teaching safety skills to children with autism spectrum disorder. Top. Lang. Disord. 27, 242-253. doi:10.1097/01.TLD.0000285358.33545.79

Sharples, S., Cobb, S., Moody, A., and Wilson, J. R. (2008). Virtual reality induced symptoms and effects (VRISE): comparison of head mounted display (HMD), desktop and projection display systems. Displays 29 (2), 58-69. doi:10.1016/j. displa.2007.09.005

Shenton, A. K. (2004). Strategies for ensuring trustworthiness in qualitative research projects. Efi 22 (2), 63-75. doi:10.3233/efi-2004-22201

Strickland, D. (1996). A virtual reality application with autistic children. Presence Teleop. Virt. Environ. 5 (3), 319-329. doi:10.1162/pres.1996.5.3.319 
Strickland, D. (1997). Virtual reality for the treatment of autism. Stud. Health Technol. Inform. 44, 81-86.

Tavassoli, T., Bellesheim, K., Siper, P. M., Wang, A. T., Halpern, D., Gorenstein, M., et al. (2016). Measuring sensory reactivity in autism spectrum disorder: application and simplification of a clinician-administered sensory observation scale. J. Autism Dev. Disord. 46 (1), 287-293. doi:10.1007/ s10803-015-2578-3

Taylor, J. L., and Mailick, M. R. (2014). A longitudinal examination of 10-year change in vocational and educational activities for adults with autism spectrum disorders. Dev. Psychol. 50 (3), 699-708. doi:10.1037/a0034297

Taylor, J. L., and Seltzer, M. M. (2011). Employment and post-secondary educational activities for young adults with autism spectrum disorders during the transition to adulthood. J. Autism Dev. Disord. 41 (5), 566-574. https://doi.org/10/czqgq4. doi:10.1007/s10803-010-1070-3

Thomas, G. (2015). How to do your case study. New York City, NY:Sage.

Thye, M. D., Bednarz, H. M., Herringshaw, A. J., Sartin, E. B., and Kana, R. K. (2018). The impact of atypical sensory processing on social impairments in autism spectrum disorder. Dev. Cogn. Neurosci. 29, 151-167. doi:10.1016/j.dcn. 2017.04.010

Tobin, G. A., and Begley, C. M. (2004). Methodological rigour within a qualitative framework. J. Adv. Nurs. 48 (4), 388-396. doi:10.1111/j.1365-2648.2004. 03207.x

Uljarević, M., Lane, A., Kelly, A., and Leekam, S. (2016). Sensory subtypes and anxiety in older children and adolescents with autism spectrum disorder. Autism Res. 9 (10), 1073-1078. doi:10.1002/aur.1602

van Steensel, F. J. A., and Heeman, E. J. (2017). Anxiety levels in children with autism spectrum disorder: a meta-analysis. J. Child Fam. Stud. 26 (7), 1753-1767. doi:10.1007/s10826-017-0687-7
Volden, J., Coolican, J., Garon, N., White, J., and Bryson, S. (2009). Brief report: pragmatic language in autism spectrum disorder: relationships to measures of ability and disability. J. Autism Dev. Disord. 39 (2), 388. doi:10.1007/s10803008-0618-y

Wang, M., and Anagnostou, E. (2014). "Virtual reality as treatment Tool for children with autism," in Comprehensive guide to autism. Editors V. B. Patel, V. R. Preedy, and C. R. Martin (Berlin: Springer), 2125-2141.

Weech, S., Kenny, S., and Barnett-Cowan, M. (2019). Presence and cybersickness in virtual reality are negatively related: a review. Front. Psychol. 10, 158. doi:10. 3389/fpsyg.2019.00158

Wong, C., Odom, S. L., Hume, K. A., Cox, A. W., Fettig, A., Kucharczyk, S., et al. (2015). Evidence-based practices for children, Youth, and young adults with autism spectrum disorder: a comprehensive review. J. Autism Dev. Disord. 45 (7), 1951-1966. doi:10.1007/s10803-014-2351-z

Yin, R. (2017). Case study research and applications: design and methods. 6th Edn. New York City, NY: SAGE.

Conflict of Interest: The authors declare that the research was conducted in the absence of any commercial or financial relationships that could be construed as a potential conflict of interest.

Copyright $\odot 2021$ Schmidt, Newbutt, Schmidt and Glaser. This is an open-access article distributed under the terms of the Creative Commons Attribution License (CC $B Y)$. The use, distribution or reproduction in other forums is permitted, provided the original author(s) and the copyright owner(s) are credited and that the original publication in this journal is cited, in accordance with accepted academic practice. No use, distribution or reproduction is permitted which does not comply with these terms. 